\title{
Quantifying the inverse spin-Hall effect in highly doped PEDOT:PSS
}

\author{
Mohammad M. Qaid, ${ }^{1}$ M. R. Mahani, ${ }^{2}$ J. Sinova, ${ }^{2,3}$ and G. Schmidt ${ }^{1,4, *}$ \\ ${ }^{1}$ Institut für Physik, Martin-Luther-Universität Halle-Wittenberg, Von-Danckelmann-Platz 3, D-06120 Halle, Germany \\ ${ }^{2}$ Institut für Physik, Johannes Gutenberg-Universität Mainz, D-55128 Mainz, Germany \\ ${ }^{3}$ Institute of Physics, Academy of Sciences of the Czech Republic, 16200 Praha, Czech Republic \\ ${ }^{4}$ Interdisziplinäres Zentrum für Materialwissenschaften, Martin-Luther-Universität Halle-Wittenberg, Nanotechnikum Weinberg, \\ Heinrich-Damerow-Strasse 4, D-06120 Halle, Germany
}

(Received 19 June 2019; revised manuscript received 19 December 2019; accepted 20 December 2019; published 25 February 2020)

\begin{abstract}
We present a systematic investigation of the inverse spin-Hall effect (ISHE) in the $\pi$-conjugated polymer poly(3,4-ethylenedioxythiophene):poly(4-styrenesulfonate) (PEDOT:PSS). Using a number of reference experiments, we are able to identify and isolate side effects which obscure the small but finite inverse spin-Hall effect in the polymer. We employ a sample geometry in which the contact areas and the area of spin current injection are laterally separated, which allows us to distinguish the ISHE from thermovoltages induced by nonreciprocal magnetostatic spin waves (MSSW) and from the ISHE induced by spin pumping through the polymer into the contacts. With an additional control experiment, we can even quantify the Nernst effect, which also needs to be taken into account. With these results, we can unambiguously show that the ISHE is present in this material, albeit at a level which requires a dedicated sample design and careful consideration of various artifacts.
\end{abstract}

DOI: 10.1103/PhysRevResearch.2.013207

\section{INTRODUCTION}

Poly(3,4-ethylenedioxythiophene):poly(4-styrenesulfonate) (PEDOT:PSS) is a conducting polymer which has been of interest for spintronics applications in past decades. The presence or absence of the inverse spin-Hall effect (ISHE) generated in YIG/PEDOT:PSS bilayer systems has been investigated in the past with contradictory results. In Ref. [1], Ando et al. claimed an effect similar in magnitude to that of YIG/Pt. In Ref. [2], our group already indicated that the so-called spin wave heat conveyor effect, which can create a temperature gradient in a typical measurement geometry for the inverse spin-Hall effect, may result in thermovoltages whose symmetry is indistinguishable in signature from the ISH voltage, even when thin-film YIG is used. In 2018, Wang et al. [3] showed that indeed the thermovoltages which appear in their sample geometry, which is similar to that used in Ref. [1], are dominating and as a result claimed that there is either no ISHE in YIG/PEDOT:PSS or that it is below their detection limit due to the strong effects of asymmetric sample heating. As we will show, it is possible to avoid the mentioned effect by using a suitable sample design. Nevertheless, we also find that further side effects need to be investigated and quantified to identify the small but finite ISHE in PEDOT:PSS.

\footnotetext{
*Corresponding author: georg.schmidt@physik.uni-halle.de
}

Published by the American Physical Society under the terms of the Creative Commons Attribution 4.0 International license. Further distribution of this work must maintain attribution to the author(s) and the published article's title, journal citation, and DOI.
The ISHE is one of the most popular mechanisms used in spintronics to detect pure spin currents $\vec{J}_{s}$ injected from a ferromagnet (FM) into a spin sink, which in most cases is nonmagnetic (NM). The injected $\overrightarrow{J_{s}}$ is caused by spin pumping either using magnetization precession $\vec{M}$ in ferromagnetic resonance (FMR) or using thermal gradients. In the case of FMR, it is described as

$$
\overrightarrow{J_{s}}=\frac{\hbar}{4 \pi} g_{\mathrm{eff}}^{\uparrow \downarrow}\left[\vec{M}(t) \times \frac{d \vec{M}(t)}{d t}\right] .
$$

Here, $\vec{M}(t)$ is the magnetization vector, $\hbar$ is the reduced Planck's constant, $g_{\text {eff }}^{\uparrow \downarrow}$ is the spin-mixing conductance for the FM/NM interface, and the spin current is polarized along $\vec{M}$ [4,5]. As shown in Fig. 2(a), the ISHE is detected as a dc voltage (or charge current $\vec{J}_{c}$ ) in the direction perpendicular to $\vec{J}_{s}$ and the spin polarization vector $\vec{\sigma}$, respectively, whose direction in the case of saturation is denoted by the external magnetic field $\vec{H}$, which orients $\vec{M}$. The relation between $\vec{J}_{c}$ and $\vec{J}_{s}$ is defined by a vector product and the spin-Hall angle $\theta_{s h}$ which describes the spin-charge conversion efficiency in the NM $[5,6]$ :

$$
\vec{J}_{c}=\frac{2 e}{\hbar} \theta_{s h}\left[\vec{J}_{s} \times \vec{\sigma}\right] .
$$

According to the $\vec{J}_{s}-\vec{J}_{c}$ conversion, $V_{\mathrm{ISHE}}$ in the perfect spin sink in contact to the FM insulator is given as [6]

$$
V_{\mathrm{ISHE}}=\frac{l \theta_{s h} \lambda_{S D} \tanh \left(d_{N M} / 2 \lambda_{S D}\right)}{d_{N M} \sigma_{N M}}\left(\frac{2 e}{\hbar}\right) J_{s},
$$

with the inverse spin-Hall voltage $V_{\text {ISHE }}$, the effective length of the area of spin pumping $l$, the spin diffusion length and the 
thickness of the spin sink $\lambda_{S D}$ and $d_{N M}$, the electron charge $e$, and the conductivity of the spin sink $\sigma_{N M}$.

In contrast to the investigation of the ISHE in metals with large spin-orbit coupling (e.g., Pt, Ta), ISHE in conducting polymers with large spin diffusion length (SDL) $[1,7]$ and high Seebeck coefficient $[3,3,8-11]$ requires special attention to side effects. From Ref. [12], it is clear that any ISHE in this material system, if existent, must be small compared to the signals measured by Ref. [1], so any attempt to measure the ISHE must take into account all physics which may cause a signal with a signature similar to that of the ISHE.

Indeed, there are a number of artifacts which can appear in the typical detection geometry for the ISHE. Only one example is nonreciprocal magnetostatic surface spin waves (MSSW) or Damon-Eshbach modes (DEM) [9,13]. Following the ferromagnetic resonance intensity, these spin waves generate a lateral temperature gradient resulting in a thermovoltage in the polymer-metal contacts which are at different respective temperatures. Because of the properties of DEM, this gradient is reversed for the opposite direction of the external magnetic field, and the resulting thermovoltage is virtually undistinguishable from the ISHE. Especially for PEDOT:PSS, the effect can be quite severe because of the large Seebeck coefficient $[8,11]$. In this case, the ISHE can only be determined either by measuring and subtracting the thermovoltage in a reference experiment or by using a special geometry which we describe below.

Using excitation by coplanar waveguides as in our experiments or in Ref. [2] certainly favors the unbalanced excitation of Damon-Eshbach modes at the top and bottom sides of the YIG film. It should, however, be noted that even for excitation in a microwave cavity [13] the asymmetric heating and the spin-wave heat conveyor effect are observed because DamonEshbach modes on the substrate side may exhibit a higher damping than at the surface.

Also in many samples, the spin source under the organic film overlaps with the metal contacts on top of the film. In this case, it is possible that a spin current flows through the polymer into the metal contacts and causes an additional ISHE there. This effect is normally discarded by using a contact material with negligible ISHE. It should be noted, however, that an ISHE negligible in one experiment may become sizable when the detection sensitivity is increased by several orders of magnitude as is typically necessary for polymers. This effect can also be avoided in the optimized geometry presented below.

A third artifact can be caused by the Nernst effect in the spin sink. At resonance condition, the absorbed power in the ferromagnet leads to a local heating which causes a perpendicular temperature gradient in the nonmagnet. Together with the external magnetic field, the resulting Nernst effect can cause a voltage which similarly to the ISHE appears only at resonance and is reversed with reversing magnetic field. Because the Nernst effect appears in the same spot as the ISHE it can only be quantified in a suitable reference experiment which allows the Nernst effect but completely excludes the ISHE. While all these effects are mostly negligible when a metallic spin sink with large spin-orbit coupling and short spin diffusion length (like Pt) is used, they can be sizable or even dominant for low spin-orbit coupling polymers. In that case, the measured voltage $V_{M}$ is composed of four components,

$$
V_{M}=V_{\mathrm{ISHE}}+V_{\mathrm{MSSW}}+V_{\text {Nernst }}+V_{\text {Contact }},
$$

where $V_{\mathrm{MSSw}}$ is the thermovoltage caused by the DEM, $V_{\text {Nernst }}$ is the voltage caused by the Nernst effect, and $V_{\text {Contact }}$ the voltage caused by spin pumping into the contacts.

\section{EXPERIMENTAL DETAILS}

\section{A. Sample fabrication}

All samples in this work use 100-nm-thick films of singlecrystal YIG with a size of $2 \times 5 \mathrm{~mm}^{2}$ grown on gadolinium gallium garnet (GGG) by liquid phase epitaxy. For a number of samples, the YIG layer is patterned to form a 2-mm-wide stripe (Fig. 4) using Ar-ion milling with $\mathrm{Al}$ foil as a shadow mask.

In order to obtain a high-quality YIG surface for increased spin pumping and ISHE [14], two subsequent cleaning procedures are performed. First, the samples are cleaned for 10 min each in acetone, methyl-isobutyl-ketone (MIBK), and isopropanol, aided by ultrasonic agitation. Then piranha solution (1:4) $\left(\mathrm{H}_{2} \mathrm{O}_{2}: \mathrm{H}_{2} \mathrm{SO}_{4}\right)$ is applied for $10 \mathrm{~min}$ followed by rinsing in deionized water for $10 \mathrm{~min}$ and drying in $N_{2}$ flow.

The PEDOT:PSS is prepared using a watery solution of $94.5 \mathrm{wt} \%$ of PEDOT:PSS (Clevios PH1000, Heraeus) doped with $5 \mathrm{wt} \%$ of dimethyl sulfoxide (DMSO) to improve the electrical conductivity and $0.5 \mathrm{wt} \%$ of Dynol as a surfactant [15]. The solution is mixed for $3 \mathrm{~h}$ using magnetic stirring. The polymer is deposited by spin coating in an ambient atmosphere at $4500 \mathrm{rpm}$ for $45 \mathrm{~s}$. Finally, the films are annealed on a hot plate at $140^{\circ} \mathrm{C}$ for $10 \mathrm{~min}$. The resulting PEDOT:PSS has a thickness of approximately $70 \mathrm{~nm}$ and an in-plane conductivity $\sigma_{\text {inplane }}=800 \mathrm{~S} / \mathrm{cm}$.

Rectangular contact electrodes of either $\mathrm{Pt}$ or $\mathrm{Ru}$ are defined at the edges of each sample. By the width of the electrodes given in Table I and the sample size also the electrode spacing is defined which is an important parameter for the analysis of the results. The electrodes are deposited by magnetron sputtering using a shadow mask to guarantee well-defined dimensions.

For control experiments, an interfacial layer of approximately $30 \mathrm{~nm} \mathrm{Al}_{2} \mathrm{O}_{3}$ is deposited on the YIG by electron beam evaporation. The layer sequence and relevant parameters for all samples investigated in this study are listed in Table I.

\section{B. ISHE characterization and setup configuration}

For the measurements, the samples are placed face down on a strip-line antenna with a thickness of $35 \mu \mathrm{m}$ and a width of $250 \mu \mathrm{m}$. While an external homogeneous magnetic field $\left(H_{e x}\right)$ saturates the magnetization of the YIG parallel to the antenna, a radio frequency (RF) current through the antenna is used to create an RF field $\left(h_{R F}\right)$ which excites the ferromagnetic resonance in the YIG. The stripline is isolated with a thin layer of PMMA to avoid any shorting between the antenna and the sample conducting layer. For the FMR spectra, the external magnetic field is modulated. The RF amplitude is measured using a diode, a lock-in amplifier, and a Keysight 34420A nanovoltmeter. ISHE measurements are carried out using copper leads attached to the Pt contacts with 
TABLE I. Samples parameters and layers dimensions for the full YIG and YIG stripe structure ( $d$, thickness; $w$, width).

\begin{tabular}{lccc}
\hline \hline Sample & YIG & $\begin{array}{c}\mathrm{Al}_{2} \mathrm{O}_{3} \\
{[\mathrm{~nm}]}\end{array}$ & $\begin{array}{c}\mathrm{Pt}[d, w] \\
{[\mathrm{nm}, \mathrm{mm}]}\end{array}$ \\
\hline S1[YIG-PEDOT:PSS-Pt] & Full & & $(10,2)$ \\
S2[YIG-Al ${ }_{2} \mathrm{O}_{3}$-PEDOT:PSS-Pt] & Full & 30 & $(10,2)$ \\
S3[YIG-PEDOT:PSS-Pt] & Full & & $(10,1)$ \\
S4[YIG-PEDOT:PSS-Pt] & Full & & $(10,2)$ \\
S5[YIG-PEDOT:PSS-Ru] & Full & & $(10,1)$ \\
S6[YIG-PEDOT:PSS-Ru] & Full & & $(10,0.5)$ \\
S7[YIG-PEDOT:PSS-Pt] & Stripe & 30 & $(10,0.5)$ \\
S8[YIG-Al $\mathrm{O}_{3}$-PEDOT:PSS-Pt] & Stripe & & \\
\hline \hline
\end{tabular}

silver glue. For these measurements the signal is detected in the same way, however, modulating the RF amplitude rather than the magnetic field. To avoid any effects by aging of the polymer, all ISHE measurements are done shortly after finishing the respective sample fabrication. The voltage values are extracted as the maximum of the curves fitted by a symmetric Lorentzian function [16].

The ISHE-voltage is given by [17]

$$
V_{\mathrm{ISHE}}=\frac{-e \theta_{s h} \lambda_{S D} \tanh \left(d_{N M} / 2 \lambda_{S D}\right) g^{\uparrow \downarrow} f l P \Theta^{2}}{d_{N M} \sigma_{N M}} .
$$

For all our samples, we can assume the same spin mixing conductance at the interface between YIG and PEDOT:PSS. In order to allow for quantitative comparison, we need to normalize by the cone angle

$$
\Theta=\frac{\gamma h_{r f}}{2 \alpha \omega},
$$

which can vary due to different damping or small variations in the coupling between the stripline antenna and the sample [12]. The necessary linewidth and RF field can be extracted from the respective FMR absorption measurements.

Furthermore, comparing different samples is only possible if the sample resistance is taken into account because the ISHE voltage is caused by the ISHE current which flows in the sample and the voltage drop is proportional to the resistance of the sample in the area where spin currents are injected. So all voltages measured are divided by the respective resistance value and only the resulting currents are plotted and compared. This normalization by the sample resistance transforms Eq. (5) to

$$
I_{\mathrm{ISHE}}=-e \theta_{S h}(w / L) \lambda_{S D} \tanh \left(d_{N M} / 2 \lambda_{S D}\right) g^{\uparrow \downarrow} f l P \Theta^{2},
$$

which is plotted for all the samples in the following, where $\lambda_{S D}, w$, and $L$ are the spin diffusion length, width, and length of the spin sink, respectively.

\section{RESULTS AND DISCUSSION}

The ISHE is measured at the uniform mode of the FMR where maximum RF absorption takes place (Fig. 1).

As our measurements show, the ISHE voltage follows the absorption of the ferromagnetic resonance. The position of the FMR line fits well the Kittel formula for the uniform FMR mode using typical material parameters for YIG, as would be expected. Nevertheless, as we have shown in Refs. [2,12], even the line which is measured for the uniform mode can have substantial overlap with low $\mathbf{k}$-vector magnon modes which are also excited during the measurement.

The first measurement is done on a sample with continuous YIG and PEDOT:PSS films, respectively, and 2-mm-wide Pt contacts (S1) [Fig. 2(a)]. This geometry is typical for ISHE measurements as in Refs. [1,3].

ISHE measurements for sample S1 are shown in Fig. 2(c). In this structure, the YIG film extends over the whole sample and thus also underneath all PEDOT:PSS and the Pt contacts. This geometry is typically used to measure the ISHE in polymers. In Ref. [1], Au contacts were used which are replaced here by $\mathrm{Pt}$ in order to allow for better identification of artifacts due to spin pumping into the contacts. As expected, the ISHE signal shows the opposite sign for the opposite magnetic field. These results are qualitatively consistent with the previous results reported for this polymer with this geometry $[1,3]$. In

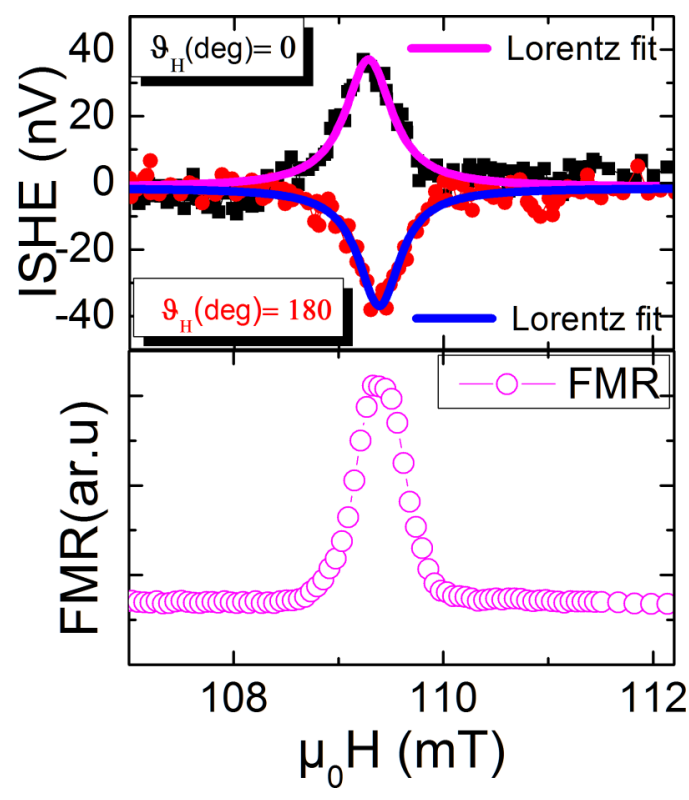

FIG. 1. ISHE at the FMR. (a) ISHE-voltage curves for YIGPEDOT:PSS bilayer structure at magnetic field angles of 0 and $180 \mathrm{deg}$ with respect to the stripline in the in-plane geometry. (b) FMR spectrum measured simultaneously to the ISHE measurement. 

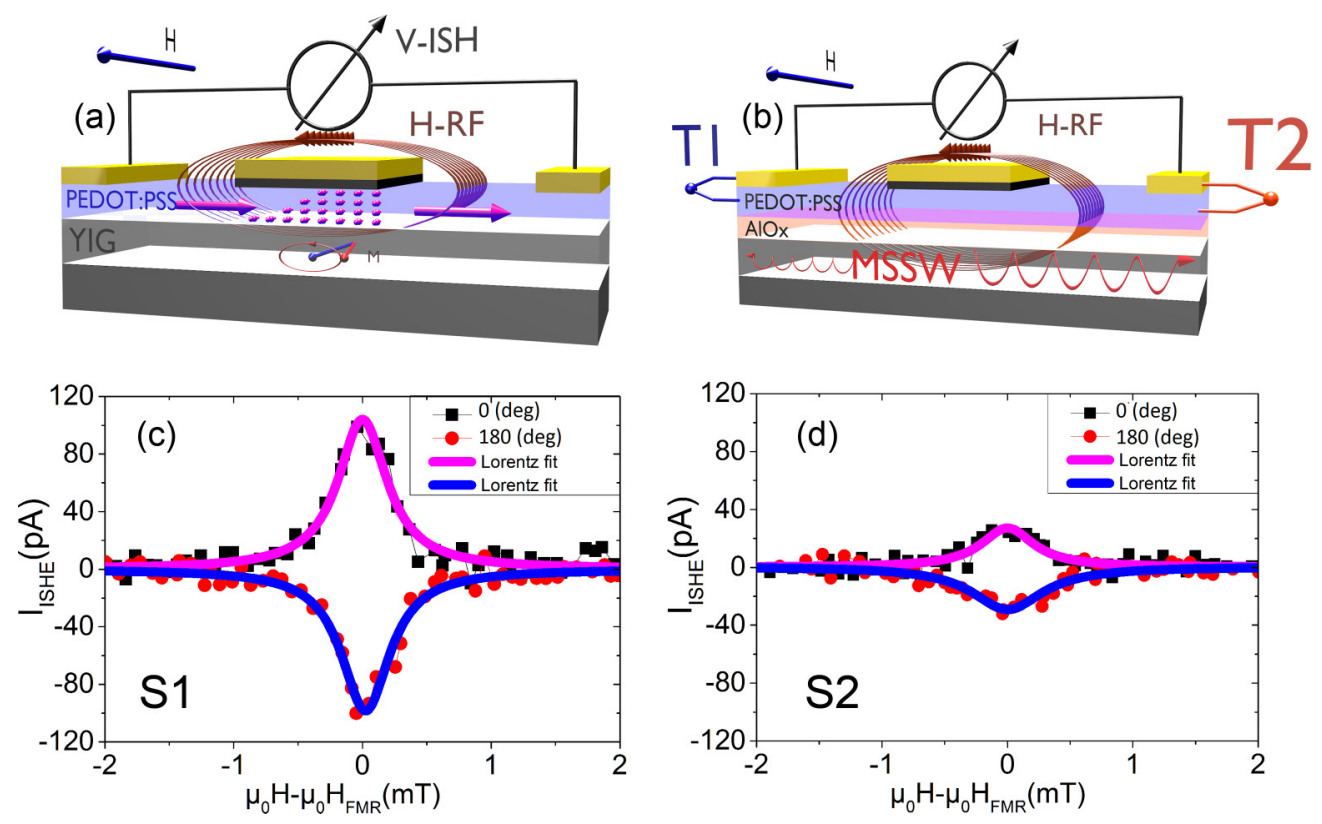

FIG. 2. ISHE current for two different YIG/PEDOT:PSS samples. Sample S1 has the geometry shown in panel (a), which is typically used in those experiments. As shown, the expectation is that the spin pumping into the PEDOT:PSS is the origin of the measured voltage. The respective signal is shown in panel (c). For sample $\mathrm{S} 2$ which has the geometry shown in panel (b), an $\mathrm{Al}_{2} \mathrm{O}_{3}$ layer prevents any spin pumping and as in Ref. [12] we expect the MSSW to create a temperature difference between the two contacts which results in a thermovoltage. The resulting signal (d) is smaller than for the sample without insulating interlayer.

Ref. [1], Ando et al. attributed this voltage to the spin-charge conversion in the polymer while Wang et al. [3] identified the thermal gradient resulting from MSSW as its origin. As we will see, both effects are present but there are even more effects to be taken into account.

To distinguish between thermally induced effects and the ISHE, we investigate a similar sample but with an insulating interlayer between YIG and PEDOT:PSS which prevents any spin pumping [Fig. 2(b)]. Structures $\mathrm{S} 2$ and $\mathrm{S} 1$ are completely identical except for a 30-nm layer of $\mathrm{Al}_{2} \mathrm{O}_{3}$ between YIG and polymer. In this case, any voltage signal must result from thermally induced effects and either be induced by the MSSW or by the Nernst effect. From experiments with YIG/Pt and $\mathrm{YIG} / \mathrm{Al}_{2} \mathrm{O}_{3} / \mathrm{Pt}$, we know that the interlayer completely suppresses the ISHE [12]. It should, however, be noted that while in the PEDOT:PSS the ISHE is now absent, the presence of the conducting polymer can still induce additional so-called radiation damping in the YIG film. In Ref. [12], we have shown that the radiation damping for materials like PEDOT:PSS can be even larger than the one induced by spin pumping and can erroneously be identified as such.

On the other hand, in Ref. [12] it was also shown that pure $\mathrm{Al}_{2} \mathrm{O}_{3}$ does not induce additional damping. This is quite important for our experiments because the asymmetric propagation of MSSW on the top and bottom side of the YIG also can be caused by different respective damping on both sides of the film. In this case, we have strongly paramagnetic GGG at the bottom and nonmagnetic $\mathrm{Al}_{2} \mathrm{O}_{3}$ with no additional damping at the top, which basically reflects the previous state of the sample without $\mathrm{Al}_{2} \mathrm{O}_{3}$.

The signals measured for S2 are shown in Fig. 2(d). Unlike the results obtained by Wang et al. [3], the ISHE signals observed here for both structures S1 and S2, respectively, are not equal but differ by a factor of 2 . This is in contrast to the assumption in Ref. [3] that the observed voltage is only a thermovoltage induced by MSSW. Because simulations show that the temperature profile in the PEDOT:PSS is not modified by the $\mathrm{Al}_{2} \mathrm{O}_{3}$ insertion, the result strongly suggests that there is at least one other effect related to spin pumping. It would, however, be premature to take this as a proof for ISHE, as we will see later.

We have investigated YIG films under similar excitation conditions using lock-in thermography in order to elucidate the lateral temperature profile. Similar measurements can be found in Ref. [2]. As a result, we find a clear temperature difference between both sides of the CPW. At the end of the structures, the temperature difference is very small if present at all and close to our detection limit of approximately $0.1 \mathrm{mK}$. However, it should be noted that the Seebeck coefficient of PEDT:PSS is sizable and even $0.1 \mathrm{mK}$ of temperature difference can lead to tens of $\mathrm{nV}$ of thermovoltage between the two contacts. It should also be pointed out that in Ref. [2] it was demonstrated that due to finite linewidth even an excitation of the uniform mode (FMR condition) has enough overlap with low $\mathbf{k}$-vector MSSW to induce a measurable temperature difference. This effect need not be present in all samples or experiments but the fact that we can observe it indicates that for a careful quantitative analysis its presence should be excluded or taken into account.

In order to investigate further side effects, we prepare a set of samples with different respective contact width $(2 \mathrm{~mm}$ in sample S4 versus $1 \mathrm{~mm}$ in sample S3). A smaller contact width simultaneously increases the spacing between the contacts from 1 to $3 \mathrm{~mm}$, modifying several aspects of the experiment. For smaller contacts, spin pumping into the contacts is reduced, as are any temperature differences between 

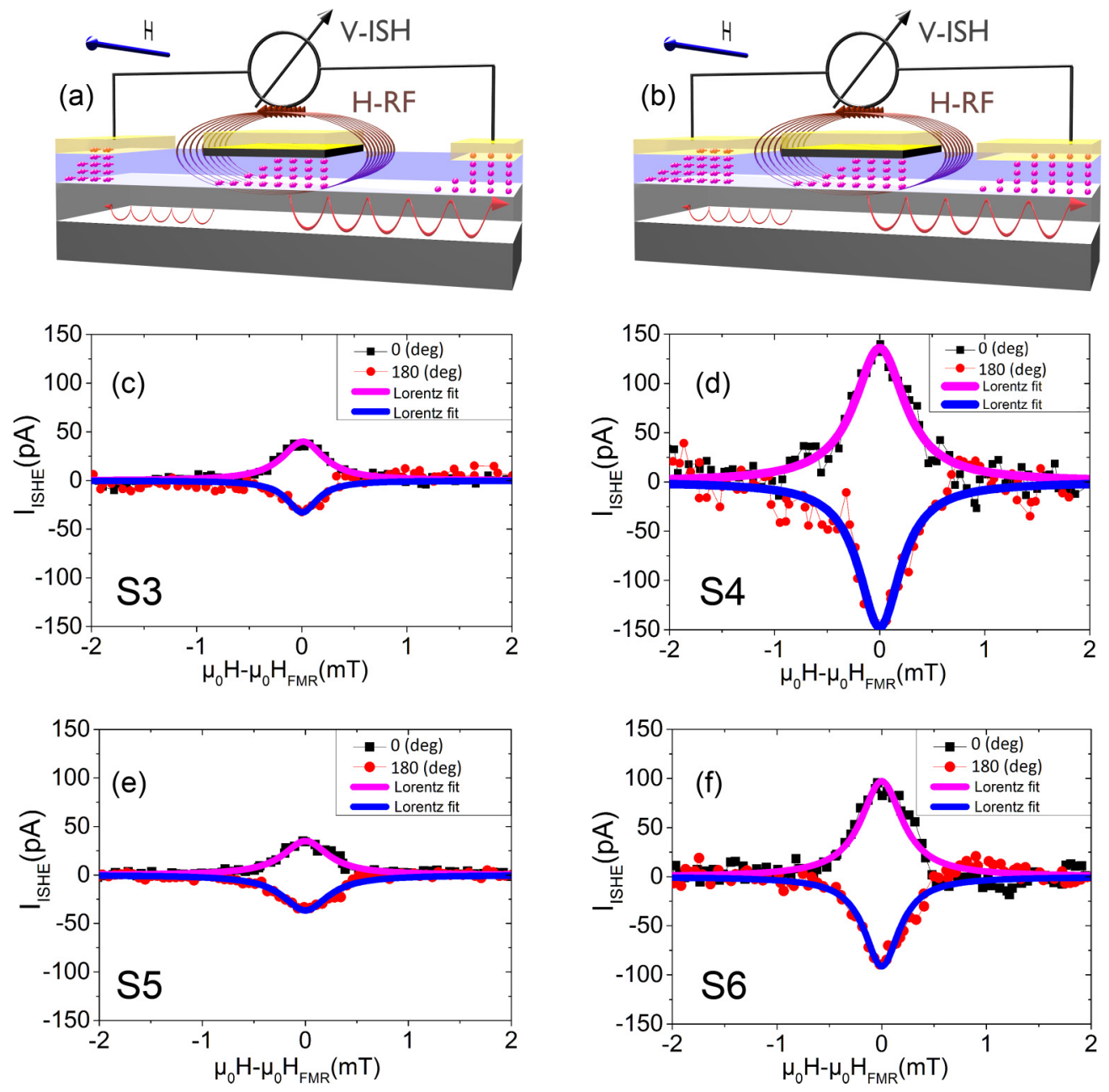

FIG. 3. ISHE results for combinations of two different respective contact widths and two different respective contact materials. As shown in panels (a) and (b), we expect the spin pumping into the contact and the related ISH voltage to cause a larger effect for wider contacts. The respective results are shown in panels (c) and (e) for 1-mm contacts and in panels (d) and (f) for 2-mm contacts. The difference between Pt $[(\mathrm{c}),(\mathrm{d})]$ and $\mathrm{Ru}[(\mathrm{e}),(\mathrm{f})]$ indicates that spin pumping into the contacts definitely contributes to the ISHE signal.

the contacts and related thermovoltages. The ISHE, however, increases because the relevant length in which the ISHE is induced is increased and shunting by the contact metal is reduced.

In our samples, smaller contacts cause a strong decrease of the signal, indicating that at least in this configuration the ISHE does not dominate [Figs. 3(c) and 3(d)]. To narrow down the origin of the signal, we investigate two more samples with 1- and 2-mm contacts, respectively, but with $\mathrm{Ru}$ as a contact material instead of Pt [Figs. 3(e) and 3(f)]. Ru has a smaller ISHE than Pt but a comparable Seebeck coefficient [18]. This allows us to distinguish between MSSW and ISHE in the contacts. Especially for the sample with 2-mm contacts, the signal is reduced by more than $25 \%$ from $\mathrm{Pt}$ to $\mathrm{Ru}$, showing that the ISHE in the contacts is not negligible although not fully responsible for the measured signal.

It should be noted that the effect can be modified and even increased by interactions between contacts and polymer during the contact deposition because the morphology may change and especially the effective polymer thickness can be reduced. We can expect that the influence of contact morphol- ogy on our analysis is minimized for the following reason: For the individual metals which were used ( $\mathrm{Pt}$ and $\mathrm{Ru}$ ), the respective samples only differ in the contact area which is in the $\mathrm{mm}^{2}$ range. Also, for $\mathrm{Pt}$ and $\mathrm{Ru}$, the deposition conditions were similar so a qualitative comparison seems adequate.

Nevertheless, a significant contribution by the MSSW is still undisputed in these samples as is also expected because of the high Seebeck coefficient of PEDOT:PSS [3,8-11].

Obviously $\mathrm{Pt}$ is not a suitable choice as a contact material if side effects are to be minimized because its large spin-Hall angle emphasizes any artifacts from spin pumping into the contacts. Nevertheless, it is useful to show that the effect can be present. As our experiments show, a different choice of material (in our case $\mathrm{Ru}$ ) may reduce the effect. However, one must always keep in mind that the ISHE that needs to be identified may also be very small and an ISHE in the contacts which compared to Pt may be negligible can be of the order of magnitude of the signal generated from ISHE in the polymer. Furthermore, there are also examples from literature where metals with larger spin-orbit coupling like $\mathrm{Au}$ [1] have been used as contact materials that can create a sizable ISH voltage. 

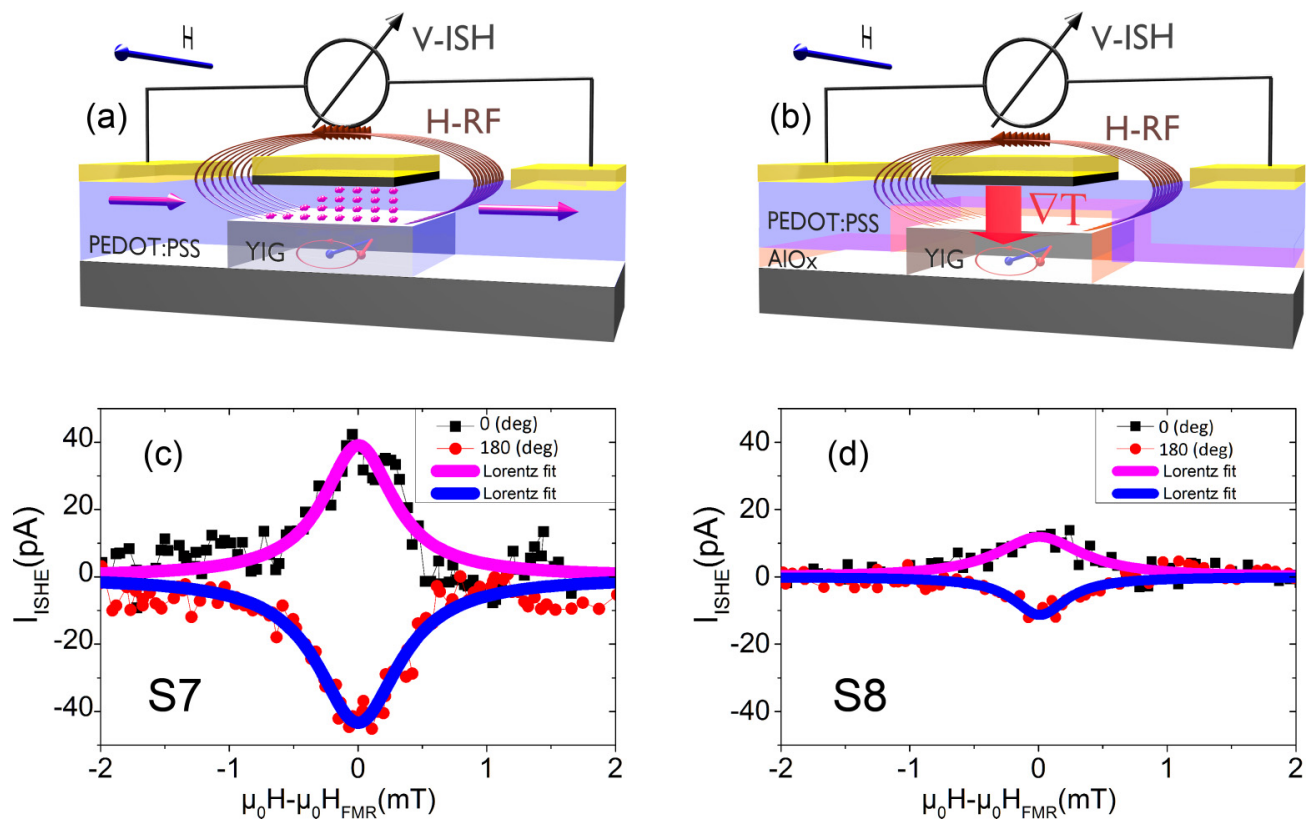

FIG. 4. Measurements of the ISHE in the optimized geometry. Sample (a) has a centered YIG stripe which does not extend underneath the contacts. The resulting ISH current is shown in panel (c). When an $\mathrm{Al}_{2} \mathrm{O}_{3}$ layer is introduced between YIG and PEDOT:PSS in this geometry, a small effect remains (d) which can be attributed to the Nernst effect caused by a vertical temperature gradient in the sample (b).

Based on these results, we have designed a sample geometry which eliminates at least the MSSW and the contact ISHE. Instead of a full YIG layer underneath the polymer, we use a YIG stripe which is smaller than and centered in the gap between the metal contacts [Fig. 4(a)]. This way no spin pumping into the contacts can take place and also the asymmetric heating of the contacts by the MSSW is dramatically reduced. It should be noted that the lateral spacing between the YIG and the contacts is of the order of $\mathrm{mm}$. Comsol simulations were performed to show that there is indeed a sizable reduction of the temperature difference at the contacts due to the new geometry. Again, we prepare one sample for the ISHE measurement (S7) and a reference sample with $\mathrm{Al}_{2} \mathrm{O}_{3}$ [S8, sample structure in Fig. 4(b)] to prevent spin pumping. As shown in Fig. 4, both samples show a finite signal; however, the signal for the sample with no spin pumping is reduced by approximately $50 \%$. These results finally prove that there is indeed an ISHE in PEDOT:PSS. Nevertheless, there is a further contribution that does not originate from the MSSW or from the contacts and that we can now attribute to the Nernst effect. The Nernst effect is caused by the vertical temperature gradient induced by the heating of the YIG, which occurs at ferromagnetic resonance. It is the only thermally generated artifact that is not avoided in our measurement geometry. To finally quantify the ISHE, the Nernst voltage needs to be subtracted from the ISHE voltage and then the ISHE must be normalized by the sample resistance which finally yields an ISHE current of approximately $25 \mathrm{pA}$.

It should be noted that a comparison between different samples based on PEDOT:PSS is not straightforward. Aging of the polymer and of the samples can change the size of the effect and it is of utmost importance to compare only samples which are based on the exact same formulations of PEDOT:PSS and solvent, ideally prepared at the same time under the same conditions and characterized immediately afterward. For our experiments, we have made sure that all samples which were compared directly were fabricated under conditions that allowed reproducibility better than the effects investigated. Sets of samples which were fabricated and measured for direct comparison constitute samples S1-S2, S3-S4, S5-S6, and S7-S8, respectively.

Because of these constraints, we have not fabricated large series of samples to get a precise number for the spin mixing conductance or the spin Hall angle. Nevertheless, theoretical calculations were performed to predict the relevant parameters for PEDOT:PSS. These parameters were used to check whether our results fit at least the order of magnitude of the expectations.

To allow for the comparison of our findings with theoretical expectations, we have made an estimate for the voltages which may be caused by the Seebeck and the Nernst effects in our geometry. In the past, Seebeck coefficients between 20 and $153 \mu \mathrm{V} / \mathrm{K}$ have been reported $[8,10,11]$. As mentioned, we expect a temperature difference of less than $1 \mathrm{mK}$ between the two contacts, which seems very small at first but for these Seebeck coefficients might cause more than $100 \mathrm{nV}$ of thermovoltage. Unfortunately, no value has been reported so far for the Nernst coefficient (N) in PEDOT:PSS. However, based on values published for other metallic-behaving polymers or simply for metals, an educated guess can be made for a Nernst coefficient of approximately $10 \mathrm{nV} / \mathrm{KT}$ $[19,20]$. To estimate the Nernst voltage, we further use $T$ (generated by the RF absorption at FMR) $=1 \mathrm{mK}, B$ (at FMR condition $)=110 \mathrm{mT}$ at $5 \mathrm{GHz}, x$ (polymer thickness $)=$ $70 \mathrm{~nm}$, and $\ell$ (effective length) $=250 \mu \mathrm{m}$. Using the formula $V_{t h}^{N}=B \ell N d T / d x$ yields $V_{t h}^{N} \sim 4 \mathrm{nV}$. Both these values are in the range of the measured voltages in our experiments, showing that they cannot be neglected from the start. 


\section{THEORETICAL CALCULATION OF SPIN-HALL $\operatorname{ANGLE}\left(\Theta_{s h}\right)$}

Our numerical calculations for the spin-Hall angle are based on a recent theoretical work on the spin-Hall effect in organics [21]. In the hopping regime, the spin-Hall effect, similar to the ordinary or the anomalous Hall effects, arises when in addition to hopping between pairs of sites (sites $\mathrm{i}$ and $\mathrm{j}$ ), the hopping in triads (hopping via an intermediate site $\mathrm{k}$ ) is also considered. The hopping integrals over a triad loop for the spin-Hall effect, give rise to a nonzero phase shift, if the molecular orientations of organic material are not aligned [21]. The importance of hopping in triads was first recognized by Holstein [22] and subsequently by others [23-30] in the study of the ordinary Hall effect and later for the anomalous Hall effect [31,32].

Here we consider a system which includes more than 10000 sites arranged on a cubic mesh. Hopping between any pairs and triads of sites within a cutoff distance is taken into account. With parameters taken from literature, we obtain the electrical conductivity by applying a small bias, within the linear response regime, across the $x$ or $y$ direction. First, the voltage drop along the $x$ or $y$ direction is obtained using

$$
\sum_{j} g_{i j}\left(V_{i}^{x / y}-V_{j}^{x / y}\right)^{2}=0,
$$

where the conductance between site $\mathrm{i}$ and $\mathrm{j}$ is defined as $g_{i j}=$ $\nu e^{-2 \alpha\left|R_{i}-R_{j}\right|} e^{-(\beta / 2)\left(\left|\epsilon_{i}\right|+\left|\epsilon_{j}\right|+\left|\epsilon_{j}-\epsilon_{i}\right|\right)}$ and $V_{i}^{x / y}$ are the potentials. The parameters $\epsilon,\left|R_{i}-R_{j}\right|, \alpha$ and $v$ are the site energies, the intersite separation, the decay constant of the localized wave functions, and the phonon frequency, respectively. The electrical conductivity is calculated by

$$
\sigma_{x / y}=\frac{1}{2 \Omega E^{2}} \sum_{j} g_{i j}\left(V_{i}^{x / y}-V_{j}^{x / y}\right)^{2},
$$

with $\Omega$ being the volume of the system. The spin-Hall conductivity is obtained via $\sigma_{s h}=J_{s}^{y} / E^{x}$ with $J_{s}^{y}$ the spin current along the $y$ direction when the electric field $E^{x}$ is applied along the $x$ direction. The spin-Hall conductivity can be written as

$$
\sigma_{s h}=-\frac{e^{2} \beta}{6 \Omega E^{2}} \sum_{j} W_{i j k}^{z}\left(V_{i j}^{x} V_{j k}^{y}-V_{i j}^{y} V_{j k}^{x}\right),
$$

where the hopping probability via an intermediate site is

$$
\begin{aligned}
W_{i j k}^{z}= & \frac{\lambda N_{i j k}^{z}}{V_{0}} \hbar v^{2} e^{-\alpha\left(R_{i j}+R_{j k}+R_{k i}\right)} \\
& \times\left[e^{-(\beta / 2)\left(\left|\epsilon_{j}\right|+\left|\epsilon_{k}\right|+\left|\epsilon_{j}-\epsilon_{i}\right|+\left|\epsilon_{k}-\epsilon_{i}\right|\right)}+i \rightleftharpoons j+i \rightleftharpoons k\right] .
\end{aligned}
$$

The spin-Hall angle is determined as $\Theta_{s h}=\sigma_{s h} /\left(\sigma_{x x} \sigma_{y y}\right)^{1 / 2}$ with $\sigma_{x x}$ and $\sigma_{y y}$ as the electrical conductivity along the $x$ and $y$ directions, respectively.

In order to ensure that the results are not the artifact of an ordered cubic mesh (not applicable for semicrystalline or disordered organics), we allow for a spatial disorder by altering the distance between any pair of sites randomly in all directions by up to $15 \%$ of its original distance. The results for the spin-Hall conductivity and the spin-Hall angle
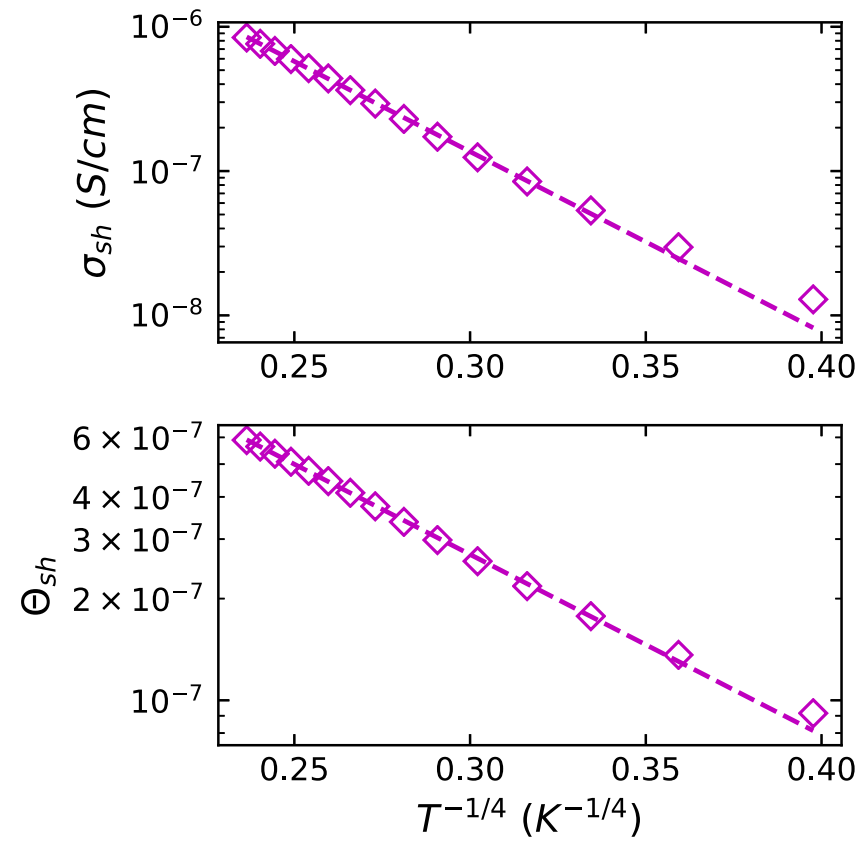

FIG. 5. Spin-Hall conductivity $\sigma_{s h}$ and spin-Hall angle $\theta_{s h}$ as a function of temperature for PEDOT:PSS.

are plotted in Fig. 5. The parameters are $\alpha=2, \lambda=10^{-3}$, $v=10^{11} \mathrm{~s}^{-1}$, and $V_{0}=0.1 \mathrm{eV}$. The transport results are consistent with variable range hopping behavior, over a temperature range $[40,320] \mathrm{K}$, as expected. At room temperature, we get $\sigma_{s h}=8 \times 10^{-7}$ and $\Theta_{s h}=6 \times 10^{-7}$, consistent with our experimental results.

Based on Eq. (7), we calculate the expected ISHE for our experiment using $\gamma=2.8 \mathrm{MH} / \mathrm{Oe}$ [12], $d=70 \mathrm{~nm}, f=$ $5 \mathrm{GHz}, l=2 \mathrm{~mm}, P=1.21$ [17] for YIG, $w=2, L=3 \mathrm{~mm}$, $h_{R F}=0.16$ Oe (corresponding to $P=80 \mathrm{~mW}$ for the given dimensions of our antenna), $\alpha=1.54 \times 10^{-4}, \lambda_{S D}=100 \mathrm{~nm}$ (based on the theoretical calculations), and $\sigma_{P E D O T: P S S}=$ $800 \mathrm{~S} \mathrm{~cm}^{-1}$. An approximate value for the spin mixing conductance of $g^{\uparrow \downarrow}=8 \times 10^{17} \mathrm{~m}^{-2}$ for YIG/PEDOT:PSS was estimated in a previous experiment [12]. The calculation yields an ISH current of $40 \mathrm{pA}$, which is nearly twice as much as we actually measure. Because of the large uncertainty of the input parameters, this is still a surprisingly good agreement with our experimental results. Nevertheless, the fact that the orders of magnitude fit confirms at least that the claim of an ISHE of this order of magnitude is credible.

\section{CONCLUSION}

We have shown that despite any previous claims [3] PEDOT:PSS exhibits a small but measurable ISHE which is in rough agreement with theory. In typical sample geometries, this ISHE is easily concealed by thermovoltages induced by MSSW or by spin pumping into the contacts. By carefully optimizing the sample geometry, it is possible to eliminate these two artifacts. The remaining small signal can be partially attributed to the ISHE and to the Nernst effect. These 
results show that extreme care is necessary to identify or even quantify ISHE in organic materials or other materials with low spin-orbit coupling. The sample geometry derived for our experiments can at least facilitate this procedure, although the Nernst effect can only be removed by careful extraction and subtraction from the original signal.

\section{ACKNOWLEDGMENTS}

This work was supported by the Deutsche Forschungsgemeinschaft (DFG, German Research Foundation) via the collaborative research center SFB 762 (TP B9) and the TRR 173-268565370; and by the ERC Synergy Grant SC2 (No. $610115)$.
[1] K. Ando, S. Watanabe, S. Mooser, E. Saitoh, and H. Sirringhaus, Nat. Mater. 12, 622 (2013).

[2] O. Wid, J. Bauer, A. Müller, O. Breitenstein, S. S. Parkin, and G. Schmidt, Sci. Rep. 6, 28233 (2016).

[3] P. Wang, L. F. Zhou, S. W. Jiang, Z. Z. Luan, D. J. Shu, H. F. Ding, and D. Wu, Phys. Rev. Lett. 120, 047201 (2018).

[4] Y. Tserkovnyak, A. Brataas, G. E. W. Bauer, and B. I. Halperin, Rev. Mod. Phys. 77, 1375 (2005).

[5] X. Tao, Q. Liu, B. Miao, R. Yu, Z. Feng, L. Sun, B. You, J. Du, K. Chen, S. Zhang et al., Sci. Adv. 4, eaat1670 (2018).

[6] K. Ando, S. Takahashi, J. Ieda, Y. Kajiwara, H. Nakayama, T. Yoshino, K. Harii, Y. Fujikawa, M. Matsuo, S. Maekawa et al., J. Appl. Phys. 109, 103913 (2011).

[7] M. Kimata, D. Nozaki, Y. Niimi, H. Tajima, and Y. C. Otani, Phys. Rev. B 91, 224422 (2015).

[8] G.-H. Kim, L. Shao, K. Zhang, and K. P. Pipe, Nat. Mater. 12, 719 (2013).

[9] T. An, V. Vasyuchka, K. Uchida, A. Chumak, K. Yamaguchi, K. Harii, J. Ohe, M. Jungfleisch, Y. Kajiwara, H. Adachi et al., Nat. Mater. 12, 549 (2013).

[10] O. Bubnova, Z. U. Khan, H. Wang, S. Braun, D. R. Evans, M. Fabretto, P. Hojati-Talemi, D. Dagnelund, J.-B. Arlin, Y. H. Geerts et al., Nat. Mater. 13, 190 (2014).

[11] N. Massonnet, A. Carella, O. Jaudouin, P. Rannou, G. Laval, C. Celle, and J.-P. Simonato, J. Mater. Chem. C 2, 1278 (2014).

[12] M. M. Qaid, T. Richter, A. Müller, C. Hauser, C. Ballani, and G. Schmidt, Phys. Rev. B 96, 184405 (2017).

[13] E. Shigematsu, Y. Ando, S. Dushenko, T. Shinjo, and M. Shiraishi, Appl. Phys. Lett. 112, 212401 (2018).
[14] S. Pütter, S. Geprägs, R. Schlitz, M. Althammer, A. Erb, R. Gross, and S. T. Goennenwein, Appl. Phys. Lett. 110, 012403 (2017).

[15] S. D. Hoath, W.-K. Hsiao, G. D. Martin, S. Jung, S. A. Butler, N. F. Morrison, O. G. Harlen, L. S. Yang, C. D. Bain, and I. M. Hutchings, J. Non-Newtonian Fluid Mech. 223, 28 (2015).

[16] E. Saitoh, M. Ueda, H. Miyajima, and G. Tatara, Appl. Phys. Lett. 88, 182509 (2006).

[17] H. L. Wang, C. H. Du, Y. Pu, R. Adur, P. C. Hammel, and F. Y. Yang, Phys. Rev. Lett. 112, 197201 (2014).

[18] M. M. Steeves, Ph.D. thesis, University of Maine, Orono, Maine, 2011.

[19] M.-S. Nam, C. Méziere, P. Batail, L. Zorina, S. Simonov, and A. Ardavan, Sci. Rep. 3, 3390 (2013).

[20] R. Fletcher, Philos. Mag. 25, 87 (1972).

[21] Z.-G. Yu, Phys. Rev. Lett. 115, 026601 (2015).

[22] T. Holstein, Phys. Rev. 124, 1329 (1961).

[23] H. Böttger and V. Bryksin, Phys. Stat. Solidi (B) 78, 9 (1976).

[24] H. Böttger and V. Bryksin, Phys. Stat. Solidi (B) 78, 415 (1976).

[25] L. Friedman, Philos. Mag. B 38, 467 (1978).

[26] P. Butcher and A. Kumar, Philos. Mag. B 42, 201 (1980).

[27] L. Friedman and M. Pollak, Philos. Mag. B 44, 487 (1981).

[28] P. Butcher and J. Mcinnes, Philos. Mag. B 44, 595 (1981).

[29] H. Böttger and V. Bryksin, Phys. Stat. Solidi (B) 113, 9 (1982).

[30] P. Butcher, J. Mcinnes, and S. Summerfield, Philos. Mag. B 48, 551 (1983).

[31] X.-J. Liu, X. Liu, and J. Sinova, Phys. Rev. B 84, 165304 (2011).

[32] J. Sinova, S. O. Valenzuela, J. Wunderlich, C. H. Back, and T. Jungwirth, Rev. Mod. Phys. 87, 1213 (2015). 